\title{
Design of 3D Visual Reservoir Modeling System Based on VB OpenGL Library
}

\author{
Rui Huang \\ College of Petroleum Engineering \\ China University of Petroleum \\ Beijing, China \\ 56805607@qq.com. \\ Xiaodong $\mathrm{Wu}$ \\ College of Petroleum Engineering \\ China University of Petroleum \\ Beijing, China \\ wuxd308@263.net
}

\author{
Ruihe Wang \\ Information Center \\ CNODC \\ Beijing, China \\ wangrh@cnpcint.com
}

\author{
Hui Li \\ Information Center \\ Richfit Information Technology Co. Ltd. \\ Beijing, China \\ lihui19@cnpc.com.cn
}

\begin{abstract}
Under the condition of $\mathrm{VB}$, the study shows it is easy to realize $3 D$ visual reservoir modeling system with the OpenGL library and grid modeling theory, which has the function of scaling, rotating, moving, multilayer interval displaying. Meanwhile, the research proposed specific ideas and case studies in order to use OpenGL library.
\end{abstract}

Keywords-reservoir modeling; $3 D$ modeling; grid; visualization; OpenGL

\section{INTRODUCTION}

3D reservoir modeling technology plays an important role in oil and gas field development technology, which is an indispensable tool for refined reservoir description and reservoir numerical simulation. Compared with VB language platform, the OpenGL library is wider used in C language class platform for realizing $3 \mathrm{D}$ visualization technique [1]. Although VB language has the shorter software development cycle, lower cost, stronger visualization, faster interface designing and other functions than $\mathrm{C}$ language [2], how to use VB language to implement this research is poor. Therefore, the research main used the OpenGL library to design 3d reservoir visual modeling system based on VB 6.0 platform in this paper.

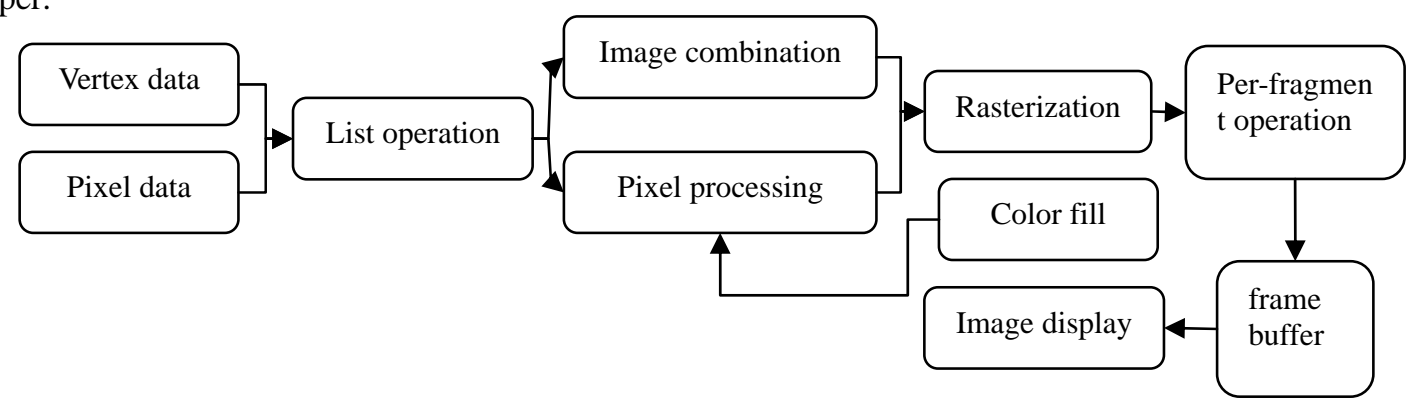

Figure 1. The workflow of VB OpenGL 3D graphics establishment

\section{The SYSTEM FUnCTION DESIGN FOR 3D VISUAL RESERVOIR MODELING SYSTEM}

Through the given coordinates of space points and grid generation theory, 3d visual reservoir modeling system establishes a 3D model based on Windows platform and VB OpenGL library. Through the human-computer

\section{The overview for VB OpenGL library}

\section{A. The introduction for VB OpenGL librarys}

OpenGL is used to build 3D model in VB6.0 by the VB OpenGL library, and the file is "vbogl.tlb". Through the TLB file, a lot of most tedious programming work can be eliminated and ultimately shorten the software development cycle effect.

\section{B. Workflow of VB OpenGL $3 D$ graphics establishment}

The steps of VB OpenGL creating 3D graphics generally include the following steps: initialization work environment, setting viewpoint, setting environment, modeling and graphics visualization. First, it must have a mathematical description of OpenGL, and based on mathematical calculation to obtain the spatial location and color information of each point on the surface of 3D object, and then rasterization, pixel processing, calculation blanking etc. Finally, delivering corresponding data body to the frame buffer[3], so the constructed 3D graphics can be carry out visual presentation in computer screen. As shown in Figure 1. interaction control[4], translation, rotation, scaling and other functions for $3 d$ reservoir model were realized, in addition, the various of effect can be displayed. In the end, the design of $3 \mathrm{~d}$ visual reservoir modeling system will be completed. As shown in Figure.2. 


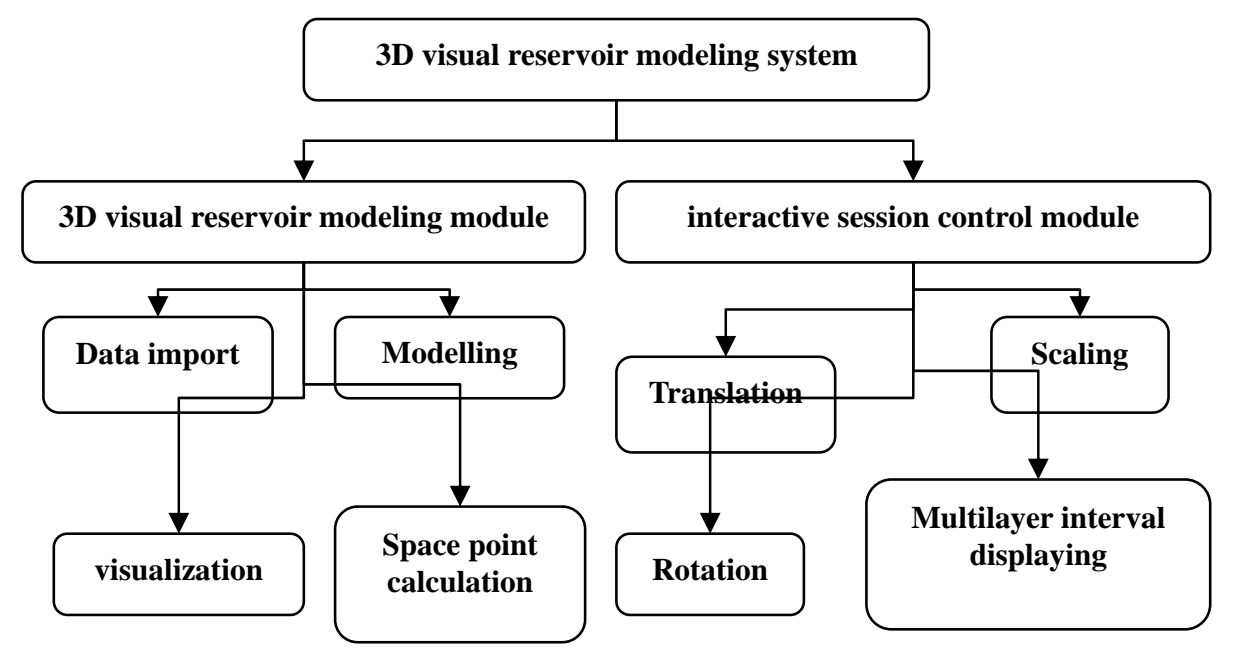

Figure 2. The system frame diagram

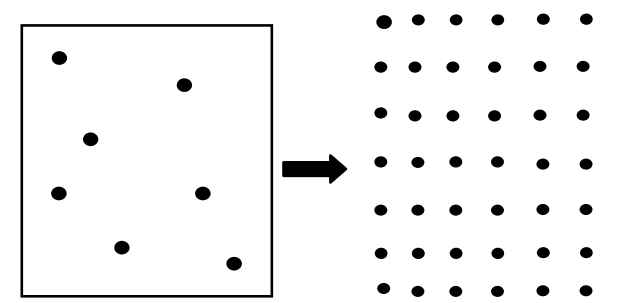

- Given point

New points

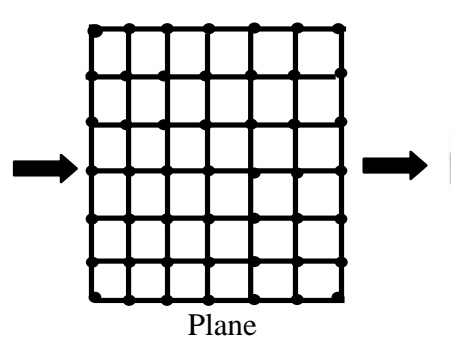

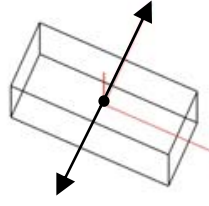

Grid unit

Figure 3. Schematic diagram of the grid generation

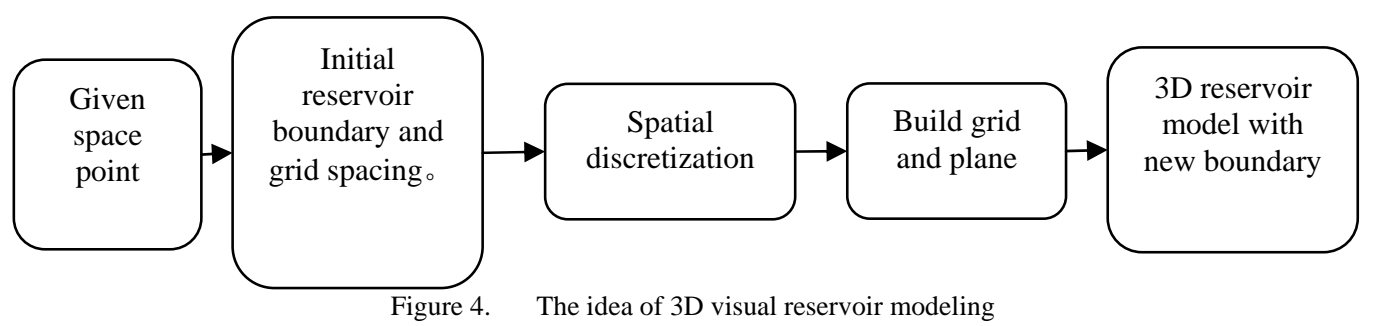

\section{KEY TECHNIQUES IN 3D VISUAL RESERVOIR MODELING SYSTEM}

\section{A. The environment of VB OpenGL library}

OpenGL is similar with state machine. It's various of modes must be set in advance so that the corresponding mode effect until modify them again. So the development environment must be built. Through initializing OpenGL graphics state and modifying the corresponding function of program, so that 3D graphics was built by OpenGL can be displayed smoothly. The main way to complete and build the environment that can support OpenGL library is select "vbogl.tlb" file through function menu "project"-"reference..." based on VB 6.0 platform, and then cite the VB OpenGL library by building the initialization function, setting the lighting mode, establishing the view and so on.

\section{$B$. The way of $3 D$ visual reservoir modeling}

3D reservoir model was generated conveniently and quickly through the space dispersed each layer of the coordinates of discrete points with the known point and the initial reservoir boundary. The 3D reservoir model was formed eventually through the theory of building grid unit from point to line, line to plane and plane to the space body.

\section{The generation of $3 D$ space coordinates}

Planar discrete points were generated based on initial rectangular reservoir boundaries. Each discrete point plane coordinates $(\mathrm{x}, \mathrm{y})$ is calculated by the equation (4.1). To gain planar new height value, we combined with the reservoir structure known space coordinates $(\mathrm{x}, \mathrm{y}, \mathrm{z})$ by using some methods such as linear interpolation, the polynomial interpolation, inverse distance weighted interpolation, kriging interpolation, spline interpolation and so on. So that the height of the new discrete points value solving realized. This article uses the method of inverse distance weighted interpolation method with constraint function for calculating a new height value to reduce amount of calculation and eliminate the interference of singular points. As the show in (4.2) 


$$
\begin{aligned}
& \left\{\begin{array}{l}
x_{i}=x_{\min }+(i-1) \cdot \Delta x, i=1,2 \cdots n+1 \\
y_{j}=y_{\min }+(j-1) \cdot \Delta y, j=1,2 \cdots m+1 \\
n=\left(x_{\max }-x_{\min }\right) / \Delta x \\
m=\left(y_{\max }-y_{\min }\right) / \Delta y
\end{array}\right. \\
& z\left(x_{i}, y_{i}\right)=\left\{\begin{array}{l}
d_{j}=\left(x_{i}-x_{j}\right)^{2}+\left(y_{i}-y_{j}\right)^{2} \\
\text { if } \cdot\left(x_{j}, y_{j}\right) \in f(d) \cdot \text { and } .\left(x_{i}, y_{i}\right) \neq\left(x_{j}, y_{j}\right) \\
\text { then } \sum_{j=1}^{c n} \frac{z_{j}}{d_{j}^{a}} / \sum_{j=1}^{c n} \frac{1}{d_{j}^{a}} \\
\text { if } .\left(x_{i}, y_{i}\right)=\left(x_{j}, y_{j}\right), \text { then } \cdot z_{j}
\end{array}\right.
\end{aligned}
$$

Etc: $\Delta x$ is the step-size of $x$ direction, $\Delta y$ is the step-size of $y$ direction, $n$ is the grid number of $x$ direction, $m$ is the grid number of $y$ direction, $f(d)$ is the discrimination function for efficient given points

V. Examples for 3D visual reservoir modeling
system

A. The $3 D$ reservoir model based on different angle of view

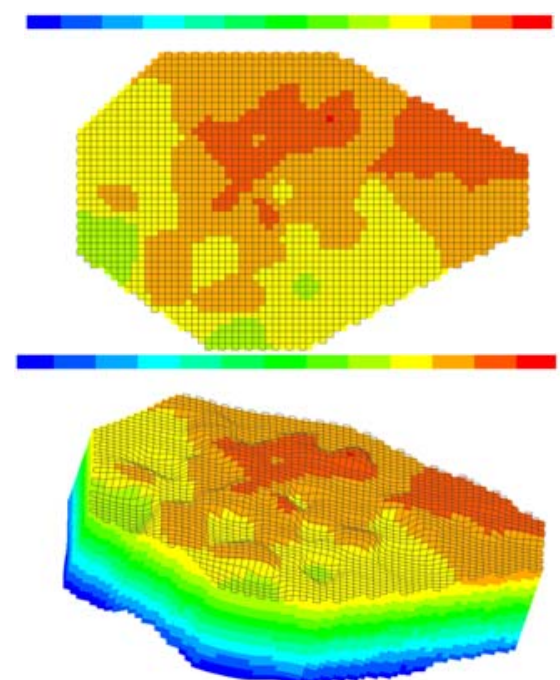

Figure.5 3D reservoir model based on different angle of view after rotating

\section{B. Multilayer interval displaying for $3 D$ reservoir model}

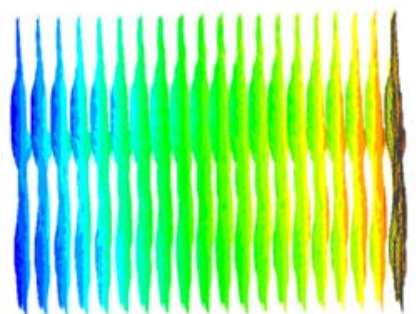

Figure.6. Multilayer interval displaying for 3D reservoir model after rotating

In these pictures different colors represent different height value, from blue to red means from small to large.

\section{CONCLUSION}

3D visual reservoir modeling system was designed based on VB OpenGL library and the grid theory, which has the function of scaling, rotating, moving, multilayer interval displaying. It can provide an important effect for researching reservoir numerical simulation and refined reservoir description.

\section{ACKNOWLEDGMENT}

The authors gratefully thank the support of Canada CMG Foundation.

\section{REFERENCES}

[1] Gao Enting, Design of 3D application based on $\mathrm{VC}++$ and OpenGL, Journal of Suzhou University(Natural Science Edition),vol.23,2007,pp.37-41.

[2] Ma Z, Zhou J. L, Liu Y .L, Design of 3-D Sporting-simulation System Based on VB and OpenGL, Coal Mine Machinery,vol.29,2008,pp.14-16.

[3] Ma J.D, Wang L.H Usage of OpenGL in the Visual Basic Environment, FOREST ENGINEERING, vol. 23,2007,pp.91-93.

[4] K.R Li, J.N Wang, K Zuo,Research on Dam Simulation System Based on OpenGL,Physics Procedia,vol.24,2012,pp.1801-1806. 